\title{
Influence of domain wall interactions on nanosecond switching in magnetic tunnel junctions
}

\author{
J. Vogel, ${ }^{1}$ W. Kuch, ${ }^{2, *}$ R. Hertel,,${ }^{3}$ J. Camarero, ${ }^{4}$ K. Fukumoto, ${ }^{2, *}$ F. Romanens, ${ }^{1}$ S. Pizzini, ${ }^{1}$ M. Bonfim, ${ }^{5}$ F. Petroff, ${ }^{6}$ \\ A. Fontaine, ${ }^{1}$ and J. Kirschner ${ }^{2}$ \\ ${ }^{1}$ Laboratoire Louis Néel, CNRS, 25 avenue des Martyrs, Boîte Postale 166, F-38042 Grenoble Cedex 9, France \\ ${ }^{2}$ Max-Planck-Institut für Mikrostrukturphysik, Weinberg 2, D-06120 Halle, Germany \\ ${ }^{3}$ Institute of Solid State Research (IFF), Research Center Jülich, D-52425 Jülich, Germany \\ ${ }^{4}$ Departamento Física de la Materia Condensada, Universidad Autónoma de Madrid, E-28049 Madrid, Spain \\ ${ }^{5}$ Departamento de Engenharia Elétrica, Universidade do Paraná, CEP 81531-990, Curitiba, Brazil \\ ${ }^{6}$ Unité Mixte de Physique CNRS/Thales, Route Départementale 128, F-91767 Palaiseau Cedex, France \\ and Université Paris-Sud, F-91405 Orsay, France
}

(Received 27 October 2005; published 6 December 2005)

\begin{abstract}
We have obtained microscopic evidence of the influence of domain wall stray fields on the nanosecond magnetization switching in magnetic trilayer systems. The nucleation barrier initiating the magnetic switching of the soft magnetic $\mathrm{Fe}_{20} \mathrm{Ni}_{80}$ layer in magnetic tunnel junctionlike $\mathrm{FeNi} / \mathrm{Al}_{2} \mathrm{O}_{3} / \mathrm{Co}$ trilayers is considerably lowered by stray fields generated by domain walls present in the hard magnetic Co layer. This internal bias field can significantly increase the local switching speed of the soft layer. The effect is made visible using nanosecond time- and layer-resolved magnetic domain imaging and confirmed by micromagnetic simulations.

DOI: 10.1103/PhysRevB.72.220402

PACS number(s): 75.60.Jk, 75.60.Ch, 75.70.-i, 85.70.Kh
\end{abstract}

The active part of devices such as spin valves and magnetic tunnel junctions, used in magnetic random access memories (MRAM), consists of an ultrathin soft ferromagnetic (FM) layer and a harder ferromagnetic layer separated by a nonmagnetic (NM) spacer layer. These devices rely on the fast switching of the magnetization of the soft layer for reading or writing separate bits of information. Micromagnetic interactions have a strong influence on this switching. Demagnetizing effects and stray fields at the edges of nanosized magnetic structures can influence the magnetic configuration and the magnetization reversal of the soft magnetic layer, but interface roughness can also play a role and induce a magnetostatic coupling with the underlying hard magnetic layer. Much larger, but more localized magnetostatic effects exist when a domain wall is present in the hard magnetic layer. ${ }^{1,2}$ Direct evidence of the influence of domain wall stray fields in one layer on the static domain configuration of another layer has been obtained by Kuch et al. ${ }^{3}$ on $\mathrm{Co} / \mathrm{Cu} / \mathrm{Ni}$ trilayers using $\mathrm{x}$-ray photoelectron emission microscopy (X-PEEM). Schäfer et al. ${ }^{4,5}$ have used Kerr microscopy to show the effect of stray fields of Bloch domain walls in an $\mathrm{Fe}$ whisker on the magnetization of a thin Fe film through a $\mathrm{MgO}$ spacer. Similar effects were recently also observed in systems with perpendicular magnetization. ${ }^{6}$ Thomas et al. ${ }^{7}$ have observed that repeated motion of domain walls in the soft magnetic layer of a FM/NM/FM trilayer can demagnetize the hard magnetic layer, even if the field used for the reversal is much smaller than the coercive field of the hard layer. In thin films with in-plane uniaxial anisotropy the static coercivity is usually determined by the field needed for domain nucleation. In FM/NM/FM trilayers, the stray field of a domain wall in the hard magnetic layer can locally decrease this quasistatic nucleation field in the soft magnetic layer. ${ }^{1}$ In this paper we show a direct, real-time observation of this effect in $\mathrm{Fe}_{20} \mathrm{Ni}_{80} / \mathrm{Al}_{2} \mathrm{O}_{3} /$ Co trilayers. In order to do so, we took advantage of the element selectivity of X-PEEM combined with $\mathrm{x}$-ray magnetic circular dichro- ism (XMCD-PEEM). Our micromagnetic simulations show that the stray field of domain walls in the Co layer locally tilts the magnetization of the $\mathrm{Fe}_{20} \mathrm{Ni}_{80}$ layer (FeNi in the following) in the direction perpendicular to the easy axis, opposite to the magnetization direction in the core of the Co domain wall. The Co domain wall stray field acts thus locally as an effective transverse bias field. ${ }^{8}$ This internal bias field decreases the nucleation barrier and can considerably increase the local switching speed of the soft layer.

The measurements were performed on a $\mathrm{Fe}_{20} \mathrm{Ni}_{80}(4 \mathrm{~nm}) /$ $\mathrm{Al}_{2} \mathrm{O}_{3}(2.6 \mathrm{~nm}) / \mathrm{Co}(7 \mathrm{~nm}) / \mathrm{CoO}(3 \mathrm{~nm}) / \mathrm{Si}(111)$ sample. The layers were deposited by rf sputtering. The Si substrate was miscut by $6^{\circ}$ along the $(\overline{2} 11)$ direction, followed by a heat treatment leading to a step-bunched surface. ${ }^{9}$ It contains terraces in the shape of ellipses, with an average length of about $1 \mu \mathrm{m}$ and a width of about $40 \mathrm{~nm}$, separated by $6-\mathrm{nm}$ high steps. The presence of $3 \mathrm{~nm}$ of $\mathrm{CoO}$ leads to an increase of the coercivity of the Co layer, which allowed doing measurements with magnetic pulses strong enough to saturate the FeNi layer without changing the Co domain pattern. The alumina layer was obtained by depositing pure $\mathrm{Al}$ followed by a glow discharge under a $10-\mathrm{Pa} \mathrm{O}_{2}$ plasma. The topographic steps in the substrate are transferred to the magnetic trilayers, ${ }^{10,11}$ leading to an in-plane uniaxial magnetic anisotropy with the easy axis along the long axis of the terraces. The steps at the end of the terraces induce a magnetostatic Néel orange-peel coupling ${ }^{11-13}$ between the two magnetic layers through the spacer layer. In Fig. 1 we show magnetization loops of the sample, obtained by longitudinal Kerr effect measurements, for fields applied both along (easy axis) and perpendicular (hard axis) to the steps. The orange-peel coupling induces a shift of the minor loops of the FeNi layer of about $1 \mathrm{mT}$ with respect to zero field. The squareness of the loops indicates that in quasistatic conditions the reversal takes place through the nucleation of one or several reversed domains and a subsequent fast propagation of the generated 


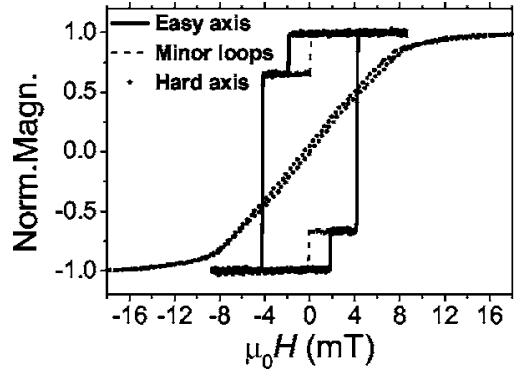

FIG. 1. Quasistatic hysteresis loops of the magnetic tunnel junctionlike trilayer obtained by longitudinal Kerr effect measurements. Loops obtained with the field applied along the easy (continuous line) and hard (dotted line) magnetization axes are shown, as well as minor loops for the FeNi layer taken along the easy axis (dashed line).

domain walls. The coercivity is determined by the nucleation barrier.

Time- and layer-resolved magnetic domain images were obtained combining XMCD-PEEM and a stroboscopic acquisition mode. ${ }^{14-16}$ We used a commercial Focus IS-PEEM microscope and an experimental setup identical to the one described in previous publications. ${ }^{17}$ Magnetic pulses provided by a small coil mounted directly on the sample were synchronized with the $\mathrm{x}$-ray photon pulses, ${ }^{14}$ with a repetition rate of 625 or $312.5 \mathrm{kHz}$. The measurements were performed on beamline UE56/2-PGM2 at the BESSY synchrotron in Berlin, Germany. The FeNi domain structure was imaged by tuning the x-ray energy to the $\mathrm{Fe} \mathrm{L}_{3}$ absorption edge $(707 \mathrm{eV})$, while for the Co layer the $\mathrm{Co} \mathrm{L}_{3}$ edge energy (778 eV) was used.

In order to study the fast switching of the FeNi layer and the influence of the Co domain walls thereon, we first induced a domain structure in the Co layer using a 3-ms pulse with an amplitude of about $10 \mathrm{mT}$. The resulting domain structure is shown in Fig. 2(1). White and black regions correspond to domains with an in-plane magnetization direction pointing parallel and antiparallel to the black arrow, respectively. Bipolar magnetic pulses with an amplitude of about 6 $\mathrm{mT}$ and a length of $40 \mathrm{~ns}$, for both the positive and the negative parts of the pulses, were then applied to the sample parallel to the easy magnetization axis. Images recorded for the FeNi layer in pump-probe mode, for different delays between photon and magnetic pulses, are shown in Figs. 2(b)-2(k). ${ }^{18}$ The corresponding positions of the photon pulses with respect to the magnetic pulses are given in Fig. 2(a). Before the pulses [Fig. 2(b)] the domain structure in the FeNi layer is strongly correlated to the one in the Co layer [Fig. 2(1)], due to the rather strong orange-peel coupling. The small overshoot at the end of the negative pulse is not sufficient to completely align the magnetization in the FeNi layer with the one in the black domain in the Co layer before the pulse. When the field increases, propagation of the domain walls in the FeNi layer takes place first [Fig. 2(c)]. At the maximum of the positive pulse some newly nucleated reversed domains also become visible [Fig. 2(d)]. At the end of the positive pulse, the FeNi layer is almost saturated except for some remaining white domains at the right and left bottom [Fig. 2(e)]. When the field is reversed, starting from this

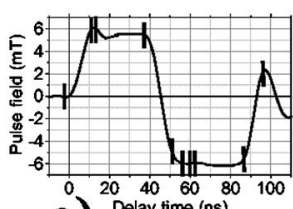

a)
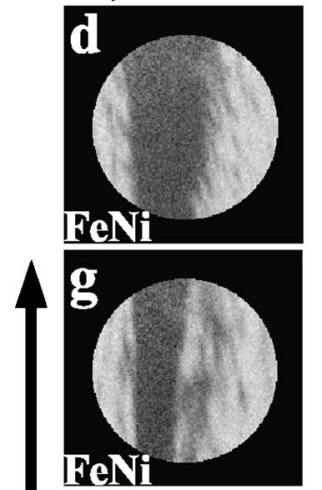

FeNi

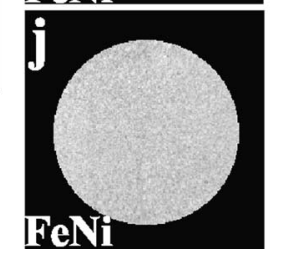

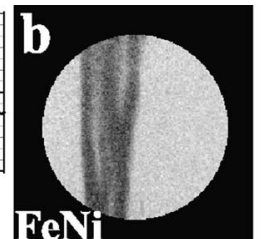

FeNi
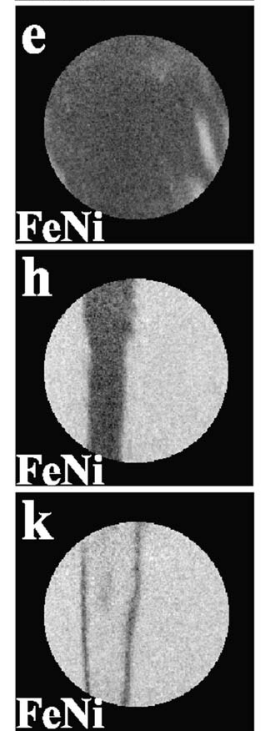
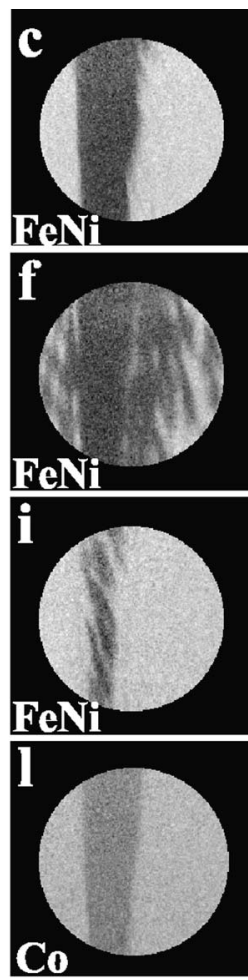

FIG. 2. Time- and layer-resolved XMCD-PEEM images of the magnetization state of the FeNi $[(\mathrm{b})-(\mathrm{k})]$ and Co layers (l). The field of view in these images is about $100 \mu \mathrm{m}$ and the spatial resolution $1 \mu \mathrm{m}$. The projection of the $\mathrm{x}$-ray incidence direction on the sample surface is pointing up in the images (parallel to the arrow) and is parallel (antiparallel) to the direction of the field for positive (negative) pulses. The magnetization direction is in the plane of the layers and points up (parallel to the arrow) for black domains, and down for white domains. The FeNi images were taken for delays between photon and magnetic pulses of $-3,11,13,37,51,56,60$, 62, 87, and $97 \mathrm{~ns}$, as indicated in (a). The Co image was taken for a delay of $60 \mathrm{~ns}$ (Ref. 18).

nearly saturated state, new white domains appear during the rising flank of the pulse [Fig. 2(f)] above the white domains in the Co layer and above the Co domain walls [Figs. 2(f) and $2(\mathrm{~g})]$. Nucleation and propagation of domain walls are faster above the white domains in the Co layer, due to the orange peel coupling leading to a higher effective field (external plus coupling fields). On the plateau of the negative pulse, the FeNi above the black Co domain switches, initially by propagation of domain walls [Fig. 2(h)] and a few nanoseconds later also by nucleation of some new white domains [Fig. 2(i)]. At the end of the negative pulse [Fig. 2(j)], the FeNi layer seems completely saturated in the white direction. Closer inspection actually shows that faint gray lines are still present at the position where domain walls are present in the Co layer. This shows that the Co domain wall stray field is strong enough to prevent saturation of the FeNi layer even for a field of $6 \mathrm{mT}$, which is about three times the static coercivity for the FeNi layer. On the other hand when the field direction is changed, preferential nucleation of reversed domains takes place above the domain wall in the Co layer, as seen in Fig. 2(k). This effect is less clear going from Figs. 2(e)-2(g) since nucleation centers become visible only after 
some time, when they have expanded through domain wall propagation to a size that is large enough to be visible with our microscope. Nucleation processes in the FeNi layer may occur on top of the Co domain walls, but at higher fields also in the middle of existing Co domains. If the field is increased very quickly to a high enough value, the corresponding critical fields are reached almost at the same time. In that case, the difference between the two nucleation fields is more difficult to see [cf. Figs. 2(f) and $2(\mathrm{~g})]$ than when the field is increased more slowly to a lower maximum value as in Fig. 2(k).

Our nanosecond time- and layer-resolved domain images thus reveal the strong influence of domain walls in the Co layer on the nanosecond reversal of the FeNi layer. We checked that for different domain patterns in the Co layer on the same spot of the sample preferential nucleation always takes place above Co domain walls. It is therefore not simply caused by topographic features. Other samples, with different miscuts and anisotropies showed the same qualitative results. This clearly indicates that the Co domain wall stray field locally decreases the barrier for nucleation of reversed domains in the adjacent FeNi layer.

The effect of stray fields of domain walls in one layer on the magnetization of the other layer in FM/NM/FM trilayers has been treated quantitatively by several authors. ${ }^{1,3,5,7,19}$ In most of these cases, the domain wall was treated as a homogeneously magnetized region of width $W_{D W}$ and with a magnetization perpendicular to the overall magnetization direction. In order to get a more precise idea of the influence of stray fields emitted by real domain walls, we have performed micromagnetic simulations using a code based on a combination of the finite element method (FEM) and the boundary element method (BEM). This FEM-BEM scheme is particularly suited to simulate magnetostatic interactions of ferromagnetic particles. ${ }^{20}$ The magnetic structures are obtained by energy minimization. For the magnetic saturation polarization $J_{S}=\mu_{0} M_{S}$ and the exchange constant $A$, values were taken of $1 \mathrm{~T}$ and $13 \mathrm{pJ} / \mathrm{m}$ for $\mathrm{FeNi}$, and $1.76 \mathrm{~T}$ and $30 \mathrm{pJ} / \mathrm{m}$ for Co, respectively. Experimentally determined values of $1720 \mathrm{~J} / \mathrm{m}^{3}$ (FeNi) and $11200 \mathrm{~J} / \mathrm{m}^{3}(\mathrm{Co})$ were used for the uniaxial anisotropy constant $K$. The magnetization was simulated in platelets with a width of $200 \mathrm{~nm}$ along the easy magnetization axis ( $y$ axis) and $400 \mathrm{~nm}$ perpendicular to this axis ( $x$ axis). To mimic extended strips, free boundary conditions along $y$ were obtained geometrically by connecting the edges of the platelet to form a ribbon shape. Two domains with opposite magnetization directions along the easy axis were introduced in the Co layer, resulting in a Néel-type domain wall. The FeNi layer in the simulations was initially homogeneously magnetized along $y$, and then its magnetization was relaxed to reach equilibrium. The results of the simulations for a 30-nm-thick spacer layer are shown in the top panels of Fig. 3. The magnetization directions are indicated with gray and black arrows for the FeNi and Co layers, respectively. In the top left panel the $x$ component of the magnetization $\left(m_{x}=M_{x} / M_{S}\right.$, with $M_{S}$ the saturation magnetization) is given, while the top right panel represents the $y$ component $\left(m_{y}=M_{y} / M_{S}\right)$. The color code ranges from blue $\left(m_{x, y}=-1\right)$ to red $\left(m_{x, y}=1\right)$ for all layers, with $m_{x, y}=0$ given by green.
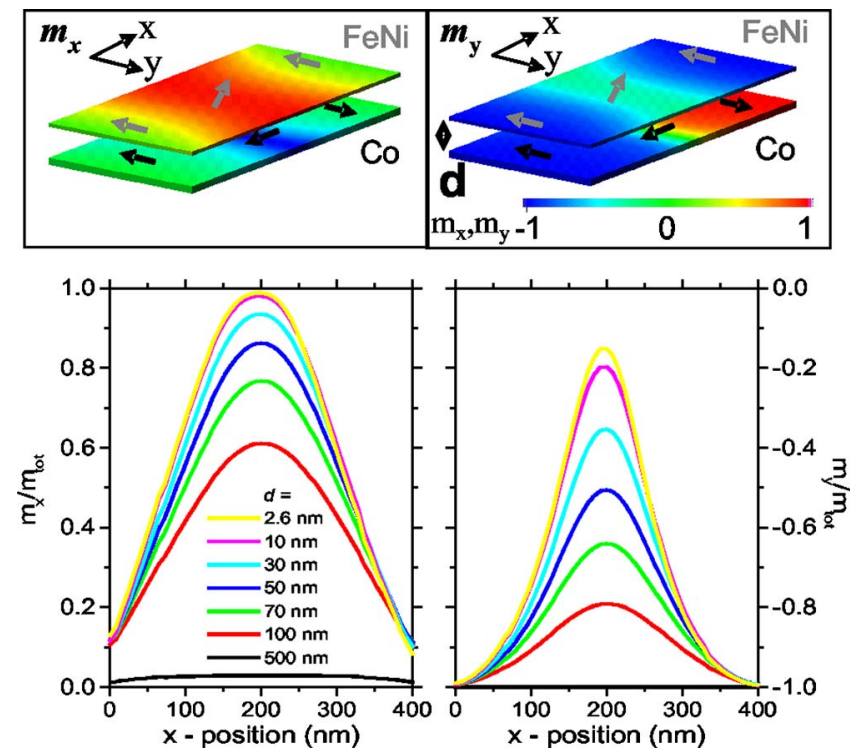

FIG. 3. (Color online) Top: Simulations of a domain wall in the Co layer and its influence on the FeNi magnetization, for a spacer thickness $d$ of $30 \mathrm{~nm}$. In the top left panel, the component of the magnetization along $x\left(m_{x}\right)$ is shown color coded for the Co (bottom) and FeNi (top) layers, while in the top right panel the $y$ component $\left(m_{y}\right)$ is given. The rectangles represent only a small fraction of the three-dimensional finite element mesh, most of which has been removed to uncover the region of interest. Bottom: $x$ component (left) and $y$ component (right) of the FeNi magnetization along $x$, in the middle of the simulated region, for different distances $d$ between the Co and FeNi layers.

The simulated width of a domain wall in the Co layer using Lilley's definition ${ }^{21}$ is about $70 \mathrm{~nm}$. The influence of the domain wall on the magnetization of the FeNi layer decreases with increasing separation between the FM layers but is considerable up to spacer thicknesses as large as $100 \mathrm{~nm}$.

The main result of the simulations is that above the Co domain wall the magnetization in the FeNi layer is strongly tilted in the direction perpendicular to the easy magnetization axis, leading to the formation of a so-called quasiwall. ${ }^{2}$ The magnetization direction in this quasiwall is opposite to the one in the center of the Co domain wall. The profile of the $x$ component of the FeNi magnetization for different separations between the two magnetic layers is shown in the bottom left panel of Fig. 3, while the $y$ component is given in the bottom right panel of Fig. 3. The $x$ component of the magnetization does not completely go to zero even at a distance of $200 \mathrm{~nm}$ of the center of the Co domain wall. The $y$ profile shows that the magnetization above the center of the Co domain wall is not perfectly perpendicular to the easy axis but makes a tilt angle $i$. This angle depends on the separation between the two layers, and a value of $81^{\circ}$ with respect to the easy axis is found for a separation of $2.6 \mathrm{~nm}$ (the experimental thickness of the alumina layer in our sample). Our simulations show that the induced magnetization angle in the FeNi layer also has an influence on the shape of the Co domain wall: it becomes wider when the interlayer distance gets smaller.

The integrated width of the region with tilted FeNi magnetization, taken from the simulated $m_{y}$ curve for a separa- 
tion of $2.6 \mathrm{~nm}$ between the layers, is about $150 \mathrm{~nm}$. From the image in Fig. 2(g) we can get an experimental estimate of this width. Taking into account the experimental resolution of $1 \mu \mathrm{m}$ and the experimental contrast, we find a value of about $250 \mathrm{~nm}$. This agrees reasonably well with the simulations, but we cannot exclude that more complicated structures, such as $360^{\circ}$ domain walls, are formed in the experiment above the Co domain walls.

In conclusion, the combination of nanosecond timeresolved XMCD-PEEM measurements and micromagnetic simulations has allowed observation and explanation of the preferential nucleation of reversed FeNi domains above Co domain walls in $\mathrm{FeNi} / \mathrm{Al}_{2} \mathrm{O}_{3} / \mathrm{Co}$ trilayers. For magnetic field pulses applied along the easy magnetization axis, the torque on the FeNi moments is very small when these are aligned antiparallel to the applied field. Above the Co domain walls, the FeNi moments are tilted away from this axis and the torque acting on them is thus much larger. The Co domain wall stray field acts as a transverse bias field that locally decreases the energy barrier for nucleation, significantly in- creasing the local switching speed. The same principle has been exploited to obtain ultrafast magnetization switching in small magnetic structures, using an external transverse bias field. ${ }^{8}$ This finding is of importance also for current-induced domain wall propagation in trilayer systems, a subject that is widely investigated nowadays because of the potential use in MRAM systems. The effect of domain wall stray fields can be controlled by manipulating the width and position of the domain wall. This allows increasing the local speed and reproducibility of magnetic switching.

We thank A. Vaurès for sample preparation. Financial support by BMBF (Grant No. 05, KS1EFA6), EU (BESSY-ECHPRI Contract No. HPRI-1999-CT-00028) and the Laboratoire Européen Associé "Mesomag" is gratefully acknowledged. J.C. acknowledges support through a "Ramón y Cajal" contract and through Project No. MAT2003-08627-C02-02 from the Spanish Ministry of Science and Technology.
*Present address: Institut für Experimentalphysik, Freie Universität Berlin, Arnimallee 14, D-14195 Berlin, Germany.

${ }^{1}$ H. W. Fuller and D. L. Sullivan, J. Appl. Phys. 33, 1063 (1962).

${ }^{2}$ A. Hubert and R. Schäfer, Magnetic Domains: The Analysis of Magnetic Microstructures (Springer-Verlag, Berlin, 1998).

${ }^{3}$ W. Kuch, L. I. Chelaru, K. Fukumoto, F. Porrati, F. Offi, M. Kotsugi, and J. Kirschner, Phys. Rev. B 67, 214403 (2003).

${ }^{4}$ R. Schäfer, R. Urban, D. Ullmann, H. L. Meyerheim, B. Heinrich, L. Schultz, and J. Kirschner, Phys. Rev. B 65, 144405 (2002).

${ }^{5}$ V. Christoph and R. Schäfer, Phys. Rev. B 70, 214419 (2004).

${ }^{6}$ S. Wiebel, J.-P. Jamet, N. Vernier, A. Mougin, J. Ferré, V. Baltz, B. Rodmacq, and B. Dieny, Appl. Phys. Lett. 86, 142502 (2005).

${ }^{7}$ L. Thomas, M. G. Samant, and S. S. P. Parkin, Phys. Rev. Lett. 84, 1816 (2000).

${ }^{8}$ B. C. Choi, M. Belov, W. K. Hiebert, G. E. Ballentine, and M. R. Freeman, Phys. Rev. Lett. 86, 728 (2001).

${ }^{9}$ M. Sussiau, F. Nguyen Van Dau, P. Galtier, A. Encinas, and A. Schuhl, J. Magn. Magn. Mater. 165, 1 (1996).

${ }^{10}$ F. Montaigne, P. Gogol, J. Briatico, J. L. Maurice, F. Nguyen Van Dau, F. Petroff, A. Fert, and A. Schuhl, Appl. Phys. Lett. 76, 3286 (2000).

${ }^{11}$ Y. Pennec, J. Camarero, J. C. Toussaint, S. Pizzini, M. Bonfim, F. Petroff, W. Kuch, F. Offi, K. Fukumoto, F. Nguyen Van Dau, and J. Vogel, Phys. Rev. B 69, 180402(R) (2004).

${ }^{12}$ L. Néel, Acad. Sci., Paris, C. R. 255, 1676 (1962).

${ }^{13}$ A. Encinas-Oropesa and F. Nguyen Van Dau, J. Magn. Magn. Mater. 256, 301 (2003).
${ }^{14}$ J. Vogel, W. Kuch, M. Bonfim, J. Camarero, Y. Pennec, K. Fukumoto, J. Kirschner, A. Fontaine, and S. Pizzini, Appl. Phys. Lett. 83, 2299 (2003); J. Vogel, W. Kuch, J. Camarero, K. Fukumoto, Y. Pennec, M. Bonfim, S. Pizzini, F. Petroff, A. Fontaine, and J. Kirschner, J. Appl. Phys. 83, 2299 (2004).

${ }^{15}$ W. Kuch, J. Vogel, J. Camarero, K. Fukumoto, M. Bonfim, Y. Pennec, S. Pizzini, A. Fontaine, and J. Kirschner, Appl. Phys. Lett. 85, 440 (2004); J. Vogel, W. Kuch, J. Camarero, K. Fukumoto, Y. Pennec, S. Pizzini, M. Bonfim, F. Petroff, A. Fontaine, and J. Kirschner, Phys. Rev. B 71, 060404(R) (2005).

${ }^{16}$ C. M. Schneider, A. Kuksov, A. Krasyuk, A. Oelsner, D. Neeb, S. A. Nepijenko, G. Schönhense, I. Mönch, R. Kaltofen, J. Morais, C. De Nadaï, and N. B. Brookes, Appl. Phys. Lett. 85, 2562 (2004); S. B. Choe, Y. Acremann, A. Scholl, A. Bauer, A. Doran, J. Stöhr, and H. A. Padmore, Science 304, 420 (2004).

${ }^{17}$ W. Kuch, R. Frömter, J. Gilles, D. Hartmann, Ch. Ziethen, C. M. Schneider, G. Schönhense, W. Swiech, and J. Kirschner, Surf. Rev. Lett. 5, 1241 (1998).

${ }^{18}$ See EPAPS Document No. E-PRBMDO-72-R03546 for the complete series (26 images) for this sample. This document can be reached via a direct link in the online article's HTML reference section or via the EPAPS homepage (http://www.aip.org/ pubservs/epaps.html).

${ }^{19}$ W. S. Lew, S. P. Li, L. Lopez-Diaz, D. C. Hatton, and J. A. C. Bland, Phys. Rev. Lett. 90, 217201 (2003).

${ }^{20}$ R. Hertel, J. Appl. Phys. 80, 5752 (2001).

${ }^{21}$ B. A. Lilley, Philos. Mag. 41, 792 (1950). 